\title{
DESTACADAS DE SOJA (GLYCINE MAX (L.) MERRIL ABSORÇÃO DE CÁlCIO E FÓSFORO POR RAÍZES VAR. IAC-2) *
}

\author{
E. Malavolta ** \\ A.F. SouZA $* * *$ \\ G.S. TUNIN *** \\ I. Chaves *** \\ J.P. DANTAS $* * *$ \\ J.F. KEFALÁS *** \\ B.F. AQUINO *** \\ J.F. FERREIRA $* * *$ \\ L.L. FOLONI *** \\ E.G.S. MOREIRA *** \\ F.A.M. LIMA *** \\ G.A. Cavalcanti $* * *$ \\ F.O.B. Mòta *** \\ E.C. VALLE $* * *$
}

\section{RESUMO}

A absorção do cálcio e do fósforo por raízes destacadas da soja var. IAC-2 foi estudada com ajuda de traçadores. Foram verificados os efeitos da concentração iônica externa do tempo, do $\mathrm{pH}$, da temperatura, da aeração e de venenos respiratórios. Os dados sugerem que a absorção do cálcio tenha se dado passivamente, sendo ativa a do fósforo. A absorção cresceu com o $\mathrm{pH}$ e a temperatura. Os valores das constantes de Michaelis encontrados concordam com os da literatura.

\section{INTRODUÇÃO}

Entre as numerosas revisões da literatura sobre a absorção iônica pelas plantas destacam-se algumas mais recentes pelo seu aspecto didático e abrangente: HIGINBOTHAM (1973), HODGES 1973), GAUSH (1972, pp. 61-175), NISSEN (1974), HELLER (1974), HEWITT E SMITH (1974, pp. 72-104), EPSTEIN (1975, pp. 73-125). E pertinente ao presente trabalho o seguinte resumo que diz respeito à absorção do cálcio e fósforo e aos fatores que nela influem.

\footnotetext{
* Com ajuda da FAPESP, CNEN, CAPES e BNDE. Entregue para publicação em 16/12/1976.

* Departamento de Química e CENA, ESALQ, USP, Piracicaba, SP

*** Estudantes de pós graduação em Solos e Nutrição de Plantas, ESALQ.
} 


\section{Teтpo}

Dentro de limites, a absorção iónica em função do tempo obedece a uma relação linear (EPSTEIN \& LEGGETT, 1954). HANDLEY \& OVERSTREET (1961) e MOORE et al., (1961) observaram que o cálcio do meio externo entrava em rápido equilíbrio com o absorvido por raízes destacadas. Ensaios de curta duração conduzidos com raízes de soja por LEGGETT \& GILBERT (1967) indicaram que uma grande parte do Ca está localizada na camada de células epidérmicas onde é fator de modificação da seletividade iônica e da retenção. LEGGETT et al., (1965) mostraram que a absorção de $\mathrm{P}$ por raízes destacadas de cevada apresentava reação linear com o tempo durante pelo menos 5 horas.

\section{Concentração iônica externa}

De um modo geral o efeito da concentração iônica externa na absorção é definido por uma hiperbole equilátera, quase sempre obedecendo à cinética michaelliana. Dependendo do elemento, uma, duas ou mais isotermas são encontradas (ver EPSTEIN, 1975, pp. 110-117). As altas concentrações externas diminuem o gradiente entre o meio externo e o interno facilitando a absorção, mas, principalmente garantindo a operação de um número maior de moléculas do carregador. HAGEN \& HOPKINS (1955) confirmaram a existência de dois sítios para a absorção do $P$. Os valores da constante de Michaellis, $\mathrm{Km}$, para o $\mathrm{H}_{2} \mathrm{PO}_{4}^{-}$variam entre $10^{-5}$ e $10^{-6}$. Em folhas de cafeeiro CRUZ (1973) encontrou $\mathrm{Km}$ da ordem de $10^{-4} \mathrm{M}$ para o Ca. SALSAC $(1966,1969)$ mostrou que, nas baixas concentrações, as raízes destacadas duma planta calcícola, Vicia faba absorvem mais $\mathrm{Ca}$ que as duma calcífuga como Lupinus luteus; em doses altas, o contrário se verifica; a velocidade de absorção, mais ou menos constante nas duas espécies até $50 \mathrm{mM}$, se eleva em seguida mais na calcífuga que na calcícola.

$$
p H
$$

O efeito direto do $\mathrm{pH}$ sobre a absorção iônica se faz sentir particularmente em valores muito baixos $(3,0-4,0)$ ou acima da neutralidade $(8,0-9,0)$ : nesses extremos diminui a absorção de $\mathrm{Ca}$ e de $\mathrm{P}$ podendo mesmo haver perda dos elementos absorvidos por desarranjos na membrana citoplasmática cuja estrutura e funcionamento são afetados. A altos valores de $\mathrm{pH}$ os íons $\mathrm{OH}^{-}$e $\mathrm{HCO}_{3}^{-}$podem competir com ânions $\mathrm{e}$, a valores baixos, pode haver competição entre $\mathrm{H}^{+} \mathrm{e}$ cátions (STEWARD \& SUTCLIFFE, 1959). 


\section{Temperatura}

Na faixa térmica $10-30^{\circ} \mathrm{C}$ há uma relação linear entre temperatura e velocidade de absorção. HANDLEY \& OVERSTREET (1961) mostraram que a $2^{\circ} \mathrm{C}$ caia consideravelmente a absorção do $\mathrm{Ca}$.

\section{Arejamento e venenos respiratórios}

Dada a dependência do processo ativo de absorção em relação à atividade respiratória como fornecedora de energia, é de se esperar diminuição na absorção iônica pela falta de aeração, pelos venenos respiratórios que bloqueiam a glicólise (como fluoreto) ou o transporte eletrônico (cianeto, sulfeto, monóxido de carbono) ou pelos desacopladores da fosforilação oxidativa (como o 2,4-dinitrofenol, DNP), o que, em geral, a experimentação confirma (EPSTEIN, 1975, pp. 92-93). MOORE et alii, 1961) verificaram que a absorção do Ca por raízes destacadas de cevada não era afetada pela temperatura e pelo DNP no $\mathrm{pH}$ 5,0 julgando-se por isso não metabólica; HANDLEY \& OVERSTREET (1961) também concluíram ser passiva a absorção do Ca em porções não vacuoladas da raiz sendo ativa naquelas contendo celulas com vacúolos. SALSAC (1969) mostrou que as curvas de absorção de Ca por Vicia e Lupinus em função do tempo atingem uma patamar rapidamente que não se modifica em 12 horas ou mais e que não é influenciado nem pelo abaixamento da temperatura e nem pela adição de DNP tudo se passando como se houvesse apenas penetração no espaço livre aparente (ELA). HELLER (1974), entretanto, aponta várias provas da absorção ativa de $\mathrm{Ca}$ em organelas (mitocôndrios e cloroplastos). CRUZ (1973) em cortes de folhas de cafeeiro verificou diminuição na absorção de Ca pelo borbulhamento com $\mathrm{N}_{2}$, pelo abaixamento da temperatura e pelo DNP pelo que conclui ser metabolicamente mediado o processo.

\section{Inibidores}

Considerando um íon qualquer, a presença de outro poderá aumentar, não modificar ou diminuir a sua absorção. O efeito inibitório poderá ser do tipo competitivo ou não competitivo.

Nos ensaios de EPSTEIN \& LEGGETT (1954) e de MENZEL \& HEALT (1955), o sódio e o magnésio não diminuiraã a absorção do Ca enquanto o Sr e o Ba fizeram-no. Para FRIED \& BROESHART (1967 a absorção do $\mathrm{Ca}^{+2}$ é inibida por $\mathrm{K}^{+}, \mathrm{Mg}^{+2}$ e $\mathrm{Ba}^{+2}$. A absorção foliar do $\mathrm{Ca}$ foi estimulada por $\mathrm{K}^{+} \mathrm{e}$ inibida não competitivamente por $\mathrm{Mg}^{+2}$ (CRUZ, 1973). LOURENÇO et al., (1968) mostraram que a absorção do P é estimulada pelo $\mathrm{Mg}$, enquanto LEGGETT et al. (1965) verificaram que Ca também o faz. Para FRIED \& BROESHART (1967) a absorção do $\mathbf{P}$ é estimulada por nitrato de cálcio. 
MATERIAL E MÉTODOS

\section{Obtenção das raízes}

As raízes de soja var. IAC-2 foram obtidas de acordo com a técnica descrita por MALAVOLTA (1975).

\section{Absorção em função do tempo}

O ensaio foi conduzido segundo MALAVOLTA (1975). Os períodos experimentais foram: 5, 10, 15, 30, 45, 60, 120 e 240 minutos.

A solução de $\mathrm{Na}^{\mathrm{H}_{2}} \mathrm{PO}_{4}$ marcada com $\mathrm{P}^{32}$ tinha a concentração de $10^{-4} \mathrm{M}$; atividade: $2 \mu \mathrm{Ci} / 100 \mathrm{ml}$ de solução.

Para estudar a absorção do $\mathrm{Ca}^{+2}$ a solução possuía uma concentração final itual a $5 \times 10^{-4}$ e $2 \mu \mathrm{Ci}$ de $\mathrm{Ca}^{45} / 100 \mathrm{ml}$.

Este, como os demais ensaios, foram feitos em duplicata; exceto quando indicado, a temperatura era da ordem de $25-30^{\circ} \mathrm{C}$.

\section{Efeito da concentração iônica externa}

Absorção do P: as concentrações usadas foram 0,01, 0,02, 0,05, 0,10, $0,20,0,50$ e $1,00 \mathrm{mM}$, sempre em presença de $\mathrm{Mg}$ como no ensaio anterior e nos demais.

Absorção do Ca: as concentrações foram as mesmas usadas no ensaio com $P$.

O período de absorção foi de 1 hora e a concentração com radioisótopos se fez como no primeiro ensaio, o mesmo acontecendo nos demais.

\section{Efeitos do $\mathrm{pH}$}

As soluções tiveram o pH ajustado a 3,0 e 6,0 empregando-se HC1 $0,1 \mathrm{~N}$ e $\mathrm{NaOH} 0,1 \mathrm{~N}$.

Período de absorção: 1 hora

\section{Efeito da temperatura}

As soluções foram mantidas a $0^{\circ} \mathrm{C}$ e a $35^{\circ} \mathrm{C}$ empregando-se respectivamente gelo e um banho de temperatura constante.

Período de absorção: 1 hora 
Efeito de veneno respiratório

Foi usado KCN numa concentração final de $5 \times 10^{-3} \mathrm{M}$. Período de absorção: 1 hora

\section{Efeito dos outros ions}

A absorção do $\mathrm{Ca}^{+2}$ foi acompanhada em presença e ausência de $\mathrm{KC} 1$ e $\mathrm{Mg} \mathrm{C} 1_{2}$ (concentração final: $10^{-2} \mathrm{M}$ ); a do $\mathrm{H}_{2} \mathrm{PO}_{4}^{-}$o foi na ausência e presença de $\mathrm{KNO}_{3}$ (concentração final $10^{-2} \mathrm{M}$ ) .

Período de absorção: 1 hora

\section{Contagem}

Terminado o período de absorção as raízes eram lavadas durante 1 minuto em água corrente e depois recebiam 3 lavagens com água destilada. Eram em seguida secas ligeiramente entre folhas de papel absorvente, transferidas para cápsulas de vidro, secas em estufa a $70-80^{\circ} \mathrm{C}$ e contadas usando-se tubo G-M de janela fina.

\section{RESULTADOS E DISCUSSÃO}

\section{Absorção em função do tempo}

Os dados encontram-se na Figura 1 que revela um comportamento diferente dos dois íons. A quantidade de $\mathrm{Ca}^{+2}$ absorvida cresce até 45 minutos aproximadamente e depois tende a se estabilizar dando um quadro idêntico ao encontrado por SALSAC (1969) e que em função de dados adicionais foi interpretado como penetração no ELA, conforme já se fez referência. Enquanto isso, a curva descritiva da absorção do $\mathrm{H}_{2} \mathrm{PO}_{4}^{-}$não mostrava tendência assintótica nem mesmo no período máximo de absorção que foi de 4 horas, indicando não saturação dos compartimentos e a operação dos mecanismos passivos e ativo, conforme era de se esperar. 


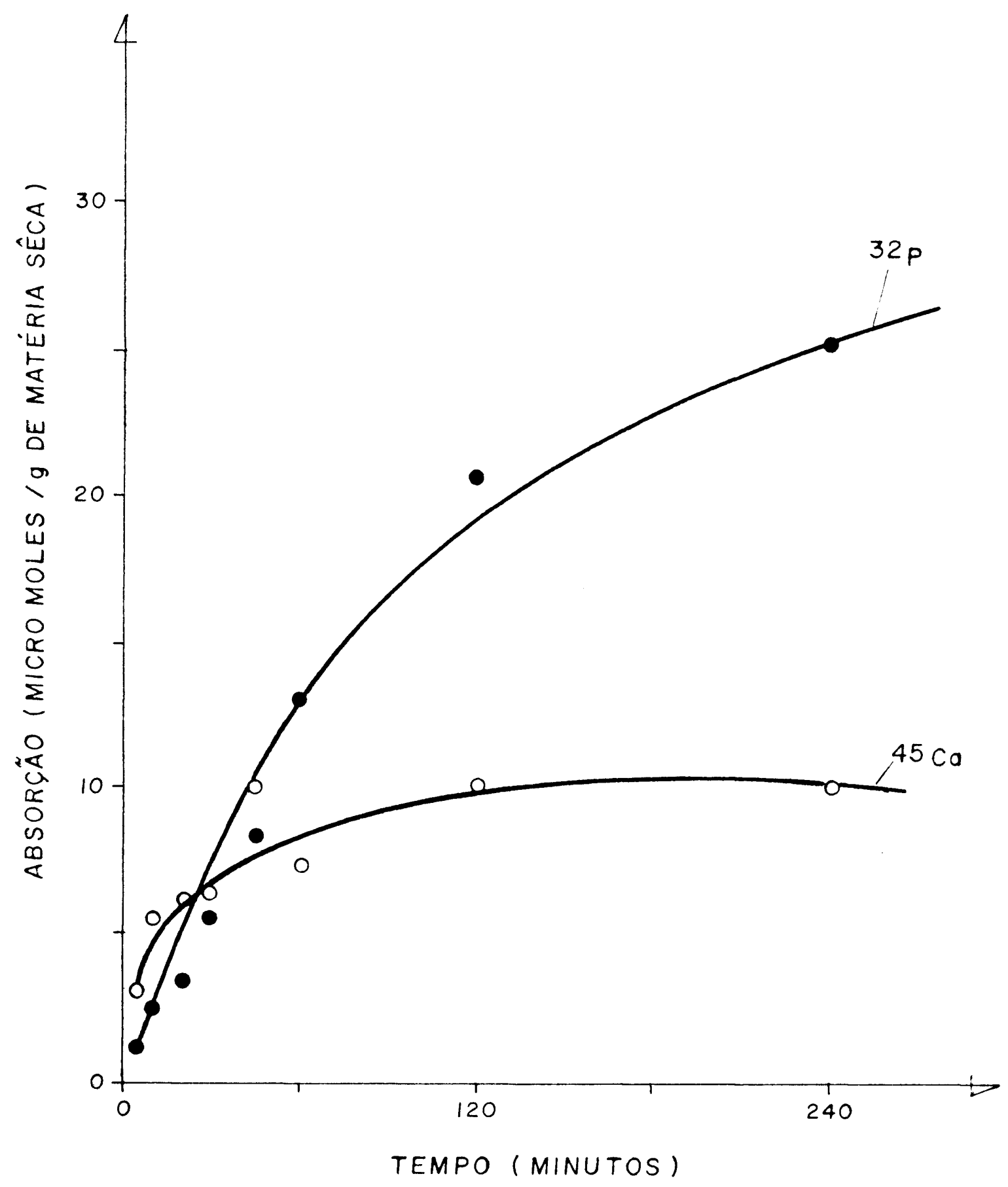

Fig. 1 - Influência do tempo na absorção do cálcio e do fósforo

\section{Concentração iônica externa}

A obediência à cinética michaeliana é aparente na Figura 2, no caso dos dois íons. O emprego da transformação de Lineawer \& Burk deu os seguintes resultados: 


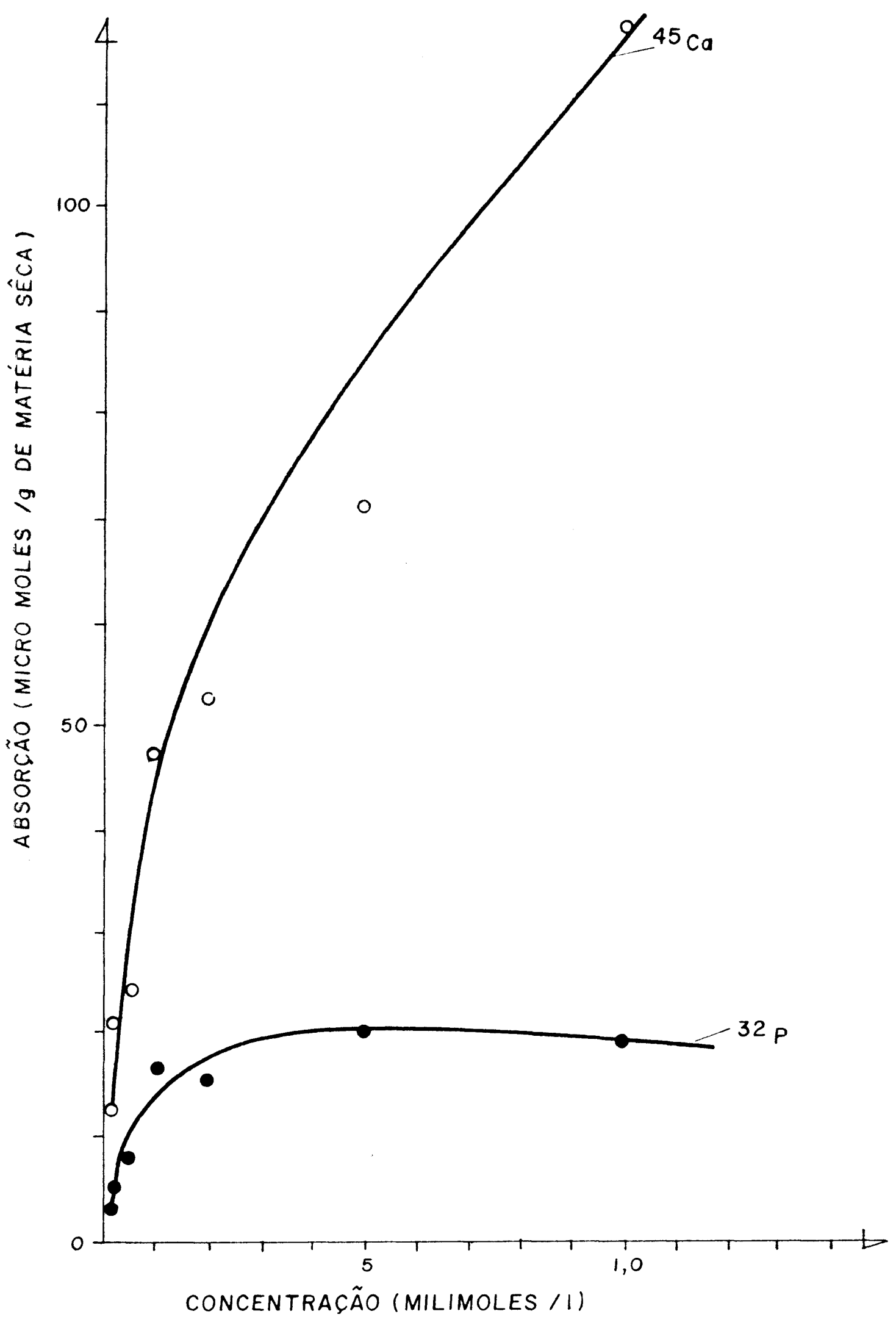

Fig. 2 - Influência da concentração iônica externa na absorção do cálcio e do fósforo

Para o $\mathrm{Ca}^{+2}-\mathrm{y}=0,022197+0,007096 \mathrm{x}$

$\mathrm{r}=0,977^{* * *}$ (significativo a $0,1 \%$ );

Para o $\mathrm{H}_{2} \mathrm{PO}_{4}^{-}=\mathrm{y}=0,103245+0,004560 \mathrm{x}$

$\mathrm{r}=0,978 * * *$ (significativo a $0,1 \%$ ). 
O alto valor de " $r$ " nos dois casos sugere a operação de mecanismos simples de absorção. Calculou-se o valor da constante de Michaelis e de Vmax, sendo encontrados:

Para o $\mathrm{Ca}^{+2}: \quad \operatorname{Vmax}=35 \mu$ moles $/ \mathrm{g} \times$ hora

$$
\mathrm{Km}=2,3 \times 10^{-4} \mathrm{M}
$$

Para $O \mathrm{H}_{2} \mathrm{PO}_{4}^{-}$: $\quad \mathrm{Vmax}=9 \mu$ moles $/ \mathrm{g} \times$ hora

$$
\mathrm{Km}=4,2 \times 10^{-5} \mathrm{M}
$$

Efeito do $\mathrm{pH}$

A Tabela 1 fornece os dados obtidos no ensaio sobre o efeito do $\mathrm{pH}$ na absorção. Vê-se que no caso dos dois íons o efeito foi significativo a $1 \%$ de probabilidade. A elevação de 3 unidades de $\mathrm{pH}$ aumentou em 7 vezes a absorção do cálcio e em 3 a do fosfato. Na verdade o efeito da elevação do pH deve ser interpretado como criação de condições favoráveis para a absorção que não existem, como se viu, em pH 3,0 conforme se lê na literatura.

\begin{tabular}{|c|c|c|}
\hline \multirow[b]{2}{*}{$\mathrm{pH}$} & \multicolumn{2}{|c|}{$\mu$ moles absorvidos / matéria seca } \\
\hline & Cálcio & Fosfato \\
\hline \multirow[t]{2}{*}{3,0} & 40,6 & 10,0 \\
\hline & 41,7 & 9.3 \\
\hline \multirow[t]{2}{*}{3,6} & 307,6 & 34,6 \\
\hline & 293,5 & 28,4 \\
\hline $\mathrm{F}$ & $1363,13^{* *}$ & $47,81 * *$ \\
\hline Dms & 30,35 & 13,7 \\
\hline $\mathrm{CV}$ & $4,1 \%$ & $15,4 \%$ \\
\hline
\end{tabular}

Tabela 1 - Efeito do pH na absorção do cálcio e do fósforo por raízes destacadas de soja

Significativo a $1 \%$

\section{Temperatura}

Em números relativos o efeito da temperatura na absorção do cálcio e do fósforo pode ser visto na Tabela 2. A análise estatística dos dados originais mostrou significância ao nível de $5 \%$ no caso do $\mathrm{P}$ e ausência de significância para a variação na absorção do Ca nos dois tratamentos. 
Tabela 2 - Efeito da temperatura na absorção do cálcio e do fósforo por raízes destacadas de soja

\begin{tabular}{ccc} 
Temperatura & Cálcio & Valores relativos \\
& Fosfato \\
\hline $0^{\circ} \mathrm{C}$ & 100 & 100 \\
$35^{\circ} \mathrm{C}$ & 394 & 112 \\
$35^{\circ} \mathrm{C} / 0^{\circ} \mathrm{C}$ & -4 & $\sim 1$ \\
\hline
\end{tabular}

\section{Venenos respiratórios}

$\mathrm{O}$ efeito do cianeto (Tabela 3 ) se fez sentir de modo significante (5\% de probabilidade) tão somente na absorção do íon fosfato que foi inibida em exatamente $50 \%$. A natureza do efeito, conhecido desde os trabalhos clássicos de LUNDEGARDH (1939) e de ROBERTSON \&

Tabela 3 - Efeito do veneno respiratório sobre a absorção do cálcio e do fósforo por raízes destacadas de soja

\begin{tabular}{lcr}
\hline \multirow{2}{*}{ Tratamento } & \multicolumn{2}{c}{ Valores relativos } \\
& Cálcio & Fosfato \\
\hline \hline $\begin{array}{c}\text { Controle } \\
+ \text { K CN }\end{array}$ & 100 & 100 \\
& 119 & 50 \\
\hline
\end{tabular}

WILKINS (1948) foi investigada há pouco por ANDERSON et al., (1974): o envenenamento por $\mathrm{CN}^{-}$, que causa forte despolarização da membrana, induz também rápido aumento na resistência da mesma; isto sugere que há na verdade um acoplamento mais direto de compostos de redox contendo ferro com a passagem facilitada de íons através da membrana; o veneno respiratório inibiria uma bomba eletrogênica de íons.

Efeito de outros ions

$\mathrm{O}$ efeito dos íons $\mathrm{K}^{+}$e $\mathrm{Mg}^{+2}$ na absorção do $\mathrm{Ca}^{+2}$ e de $\mathrm{NO}_{3}{ }^{-}$na do fosfato podem ser vistos na Tabela 4, usando-se números índices. 
Tabela 4 - Efeito da presença de outros íons na absorção do cálcio e do fósforo por raizes destacadas de soja

\begin{tabular}{lcc}
\hline \multirow{2}{*}{ Tratamento } & \multicolumn{2}{c}{ Absorção relativa } \\
\hline \hline Controle & Cálcio & Fosfato \\
$+\mathrm{K}^{+}$ & 100 & 100 \\
$+\mathrm{Mg}^{+2}$ & 120 & - \\
$+\mathrm{NO}_{3}^{-}$ & 79 & - \\
\hline
\end{tabular}

A análise estatística mostrou significância (a 5\%) apenas para o efeito inibitório do $\mathrm{Mg}^{+2}$ na absorção do cálcio. Embora os dados não permitam a conclusão, é atraente sugerir que se trata de inibição do tipo competitivo dada a semelhança dos dois íons.

\section{RESUMO E CONCLUSÕES}

Foram conduzidos experimentos com raízes destacadas de soja var. IAC-2, destinados a esclarecer a natureza do(s) processo(s) de absorção de cálcio e de fósforo e a estudar os fatores externos que a influenciam, tendo sido tiradas as seguintes conclusões principais:

(1) a absorção do cálcio não segue a cinética michaeliana usual, a evidência sendo a de operação de mecanismo(s) passivo(s);

(2) a absorção do fósforo é metabólica, ao que parece havendo apenas um mecanismo (ou carregador) em ação;

(3) o aumento do $\mathrm{pH}$ de 3,0 para 6,0 tem acentuado efeito na absorção dos dois íons;

(4) baixa temperatura e veneno respiratório inibiram a absorção do fósforo mas não a do cálcio;

(5) o íon magnésio diminuiu a absorção do cálcio mas o potássio não teve efeito; o íon nitrato não influenciou a absorção do fosfato. 


\section{SUMMARY}

\section{ABSORPTION OF CALCIUM AND PHOSPHORUS BY EXCISED SOYBEAN ROOTS, VAR, IAC-2}

This paper deals with experiments designed to study the influence of several external factors on the uptake both of $\mathrm{Ca}^{+2}$ and $\mathrm{H}_{2} \mathrm{PO}_{4}^{-}$by excised soybean roots.

The main conclusions are as follows:

1) Ca uptake does not follow the usual Michaelian kynetics, the evidence suggesting the operation of passive mechanism;

2) $P$ uptake is metabolic, a single mechanism being in operation;

3) $\mathrm{pH}$ has a strong effect both on $\mathrm{Ca}$ and $\mathrm{P}$ absorption;

4) The use both of lower temperature $\left(\mathrm{O}^{\circ} \mathrm{C}\right)$ and of respiratory poisons (cyanide) inibited phosphate uptake but had no effect on calcium absorption.

5) The obsorption of $\mathrm{Ca}^{+2}$ was lowered in the presence of $\mathrm{Mg}^{+2}$ but not in that of $\mathrm{K}^{+}$; nitrate ions had no detrimental or sinergistic offect on the absorption of phosphate.

\section{LITERATURA CITADA}

ANDERSON, W.P.; D.L. HENDRIX \& N. HIGINBOTHAM. 1974 - The effect of cyanide and carbon monoxide on the electrical potential and resistance of cell membranes. Plant Physiol. 54:712-716.

CRUZ, A.D. 1973 - Absorção do cálcio por tecidos da folha do cafeeiro (Coffea arabica L., var. Mundo Novo). Tese de Doutoramento, ESALQ, Piracicaba.

EPSTEIN, E. 1975 - Nutrição Mineral das Plantas - Princípios e Perspectivas. Trad. e notas de E. Malavolta. Livros Técnicos e Científicos Editora S.A. e Editora da USP, São Paulo.

EPSTEIN, E. \& J.E. LEGGET. $1954 \rightarrow$ The absorption of alkaline earth cations by barley roots: kinetics and mechanism. Amer. J. Bot. 41 :785-791.

FRIED, M. \& H. BROESHART. 1967 - The Soil Plant System. Academic Press. Nova Iorque e Londres.

GAUCH, H. 1972 - Inorganic Plant Nutrition. Dowden, Hutchinson \& Ross, Inc. Stroudsburg, P.

HAGEN, C.E. \& H.T. HOPKINS. 1955 - Ionic species in orthophosphate absorption by barley roots. Plant Phisiol. $30: 192-199$.

HANDLEY, R. \& R. OVERSTREET. 1961 - Uptake of calcium and chlorine in roots of Zea mays. Plant Physiol. $36: 766-769$. 
HELlER, R. 1974 - L'Absorption Minérale ches les végétaux. Masson \& Cie., Paris.

HEWITT, E.J. \& J.A. SMITH, 1974 - Plant Mineral Nutrition. The English Universities Press Ltd., Londres.

HigINBOTHAM, N. 1973 - The mineral absorption process in plants. The Bot. Rev. $39(1): 15-69$.

HODGES, T.K. 1973 - Ion absorption by plant roots. Adv. Agron. 25 :163-207.

LEGGET, J.R. \& W.A. GILBERT. 1967. - Localization of the Ca mediated apparent ion seletivity in the cross sectional volume by soybean roots. Plant Physiol. 42:1658-1664.

LEGGETT, J.E., R.A. GALLOWAY \& H.C. GAUCH. 1965 -.. Calcium activation of orthophosphate absorption by barley roots. Plant Physiol. 40:897-902.

LOURENÇO, S., O.J. CROCOMO, I.R. NOGUEIRA \& E. MALAVOLTA. 1968 Kinetic studies of phosphorus uptake by exccesed roots of barley in the presence of magnesium. An. Acad. Bras. Ciênc. 40(2):171-179.

LUNDEGARD, H. 1939 - An electro-chemical theory of salt absorption and respiration. Nature. 143: 203.

MALAVOlTA, E. 1975 - Prática's de Nutrição Mineral de Plantas. Postila mimeo., Piracicaba.

MENZEL, R.G. \& W.R. HEALD. 1955 - Distribution of potassium, rubidium, caesium calcium and strontion within plants grown in nutriente solution. Soil Sci. 80:287-293.

MOORE, D.P., L. JACOBSON \& R. OVERSTREET. 1961 - Uptake of calcium by excised barley roots. Plant. Physiol. 36:53-57.

NISSEN, P. 1974 - Uptake mechanisms inorganic and organic. Ann. Rev. Plant Physiol. $25: 53-79$.

ROBERTSON, R.N. \& N.J. WILKINS. 1948 - Studies on the metabolism of plant cells. VII. The quantitative relation between salt accumulation and salt respiration. Aust. J. Sci. Res. B. Biol. Sci. $1: 17-37$.

SALSAC, L. 1966 - Absorption du calcium dans les racines de Lupin Jaune et de Féverole. C.R. Acad. Sci., Paris. $262: 2462-2465$.

SALSAC, L. 1969 - Caracteres particuliers de l'absorption du calcium. Bull. Soc. Franc. Physiol. vég. $15: 213-236$.

STEWARD, F.C. \& J.F. SUTCIJFFE. 1959 - Plants in relation to inorganic salts. Plant Physiology. II, pp. 253-478, Academic Press, Nova Iorque e Londres. 